\title{
Operation of a Diamond Cryogenic Detector for Low-Mass Dark Matter Searches
}

\author{
L. Canonica ${ }^{1}$ (D) - A. H. Abdelhameed ${ }^{1} \cdot$ P. Bauer $^{1} \cdot$ A. Bento ${ }^{1,2} \cdot$ E. Bertoldo ${ }^{1}$. \\ N. Ferreiro lachellini ${ }^{1}$. D. Fuchs ${ }^{1} \cdot$ D. Hauff ${ }^{1} \cdot$ M. Mancuso ${ }^{1} \cdot$ F. Petricca ${ }^{1}$. \\ F. Pröbst ${ }^{1} \cdot$ J. Rothe ${ }^{1}$
}

Received: 6 August 2019 / Accepted: 14 January 2020 / Published online: 1 February 2020

(c) The Author(s) 2020

\begin{abstract}
Despite the multiple and convincing evidence of the existence of dark matter (DM) in our Universe, its detection is one of the most pressing questions in particle physics. As of today, there is no unambiguous hint which could clarify the particle nature of DM. For these reasons, a huge experimental effort is ongoing, trying to realize experiments which can probe the particle properties of DM. In particular, direct search experiments are trying to cover the largest possible mass range, from a few MeVs up to TeVs. Particularly suited for the sub-GeV mass region are detectors containing light nuclei, which are sensitive to the scattering of light DM candidates. Among them, we investigate a carbon-based absorber to explore DM masses down to the $\mathrm{MeV}$ region. Thanks to their cryogenic properties (high Debye temperature and long-lived phonon modes), carbon-based materials operated as low temperature calorimeters could reach an energy threshold in the eV range and would allow for the exploration of new parameters of the DM-nucleus cross section. Despite several proposals, the possibility of operating a carbon-based cryogenic detector is yet to be demonstrated. In this contribution, the preliminary results obtained with a diamond absorber operated with a TES temperature sensor will be reported. The potential of such a detector in the current landscape of DM searches will also be illustrated.
\end{abstract}

Keywords CVD diamond · Dark matter · Cryogenic detector · TES

L. Canonica

canonica@mpp.mpg.de

1 Max-Planck-Institut für Physik, Föhringer Ring 6, 80805 Munich, Germany

2 Departamento de Fisica, Universidade de Coimbra, P3004 516 Coimbra, Portugal 


\section{Introduction}

From the observation of galaxies to the measurement of the cosmic microwave background, several pieces of evidence suggest that the Universe is filled by the nonluminous dark matter (DM). Many particle candidates for DM have been proposed, but none of them has been detected yet. As a consequence, the experiments have just set upper limits in the region of parameters described by the cross section for the DM-nucleus interaction and the mass of the DM particle. In the absence of a positive detection, in the recent years a large fraction of the scientific community started to explore the possibility of detecting DM with mass in the sub-GeV range. This is supported by well motivated theories where light DM particles arise from hidden sectors (see [1-3] and references therein).

Among the different experimental approaches, cryogenic detectors are well suited for the search of light DM interactions with target nuclei. Experiments using cryogenic detectors (CRESST [4], EDELWEISS [5] and SuperCDMS [6]) are leading the sensitivity in the low-mass region for DM-nucleus interactions. In such experiments, the elastic scattering of DM particles with the nuclei of the target would produce a nuclear recoil that is detected as a temperature variation of the cryogenic sensor. For the detection of sub-GeV DM particle, a light target nucleus is kinematically preferable, compared to heavier target nuclei. Carbon represents an ideal choice for a low-mass DM detector, and diamond in particular for its excellent intrinsic thermal properties. In the following sections, the properties of diamond as cryogenic target for low-mass DM detection will be described and the results of the first operation at cryogenic temperature of a diamond absorber coupled to a transition edge sensor (TES) will be presented.

\section{Diamond as Low-Mass DM Detector}

The kinematic of the DM direct detection is described in terms of the masses of the target material and of the incoming DM particle. When a DM particle with energy $E_{\mathrm{DM}}$ and mass $m_{\mathrm{DM}}$ elastically and coherently scatters off a target nucleus of mass $m_{\mathrm{T}}$, it will deposit the recoil energy $E_{\mathrm{R}}$ :

$$
E_{\mathrm{R}}=E_{\mathrm{DM}} \frac{2 \mu^{2}}{m_{\mathrm{DM}} m_{\mathrm{T}}}(1-\cos \theta)
$$

where $\mu$ is the reduced mass of the nucleus-DM system $m_{\mathrm{DM}} m_{\mathrm{T}} /\left(m_{\mathrm{DM}}+m_{\mathrm{T}}\right)$ and $\theta$ is the scattering angle in the center of mass reference frame.

It is then clear that the composition of the target plays a crucial role in the capability of a given material to investigate different parameters in the DM-nucleus interaction landscape. In particular, for a DM particle much lighter than the mass of the target nucleus we have $\mu \sim m_{\mathrm{DM}}$ and the recoil energy $E_{\mathrm{R}}$ scales with $m_{\mathrm{DM}}^{2}$. This shows that an extremely low energy threshold is needed to detect sub-GeV DM. The state-of-the-art technology has successfully demonstrated the possibility to operate cryogenic detectors with thresholds of a few tens of eV (in $\mathrm{CaWO}_{4}$ [4], Ge [5], and 
$\mathrm{Al}_{2} \mathrm{O}_{3}$ [7]), using TES and neutron transmutation-doped (NTD) Germanium thermistors. If such a threshold could be obtained also with a diamond target, this would become an excellent candidate material for the detection of DM in the next years.

Diamond represents an ideal material for the detection of low-mass DM particles. It is made of a light element $(Z=6)$, and with a Debye temperature of $2220 \mathrm{~K}$, this material is characterized by high-energy and long-lived phonon modes, which should result in optimal performance as cryogenic detector. Moreover, diamond is also a semiconductor, with excellent isotopic purity. This makes a simultaneous readout of the phonon and the charge signals possible, allowing for background discrimination (as performed by the experiments described in Refs. [5, 6]). A complete review of the properties of diamond as cryogenic sub-GeV DM detector can be found in [8]. The expected nuclear recoil rate for different masses of DM particles is shown in Fig. 1. For lower DM masses, the recoil spectrum is compressed toward lower energies and the threshold is the only key issue for reaching the sensitivity in the sub-GeV range. Despite the excellent properties of diamond as a target, it has never been operated as a cryogenic detector with TES sensor until today. In the early 90s, a $100 \mathrm{mg}$ diamond operated with an NTD showed an energy resolution of $3 \mathrm{keV}$ FWHM (at $80 \mathrm{keV}$ ) [9]. It is clear that a diamond detector with much lower threshold could be the ideal tool to explore sub-GeV DM candidates. One of the main obstacles that still prevents a large usage of this material in the scientific community is its cost, together with the high purity that is needed to operate a crystal as cryogenic detector. Nevertheless, in the last few years, thanks to the use of diamonds in the semiconductor electronics industry, the cost of high-purity diamonds has dropped and has become now more accessible for a variety of research fields. Among them, the quantum computing community is focusing its attention on lattice vacancies in

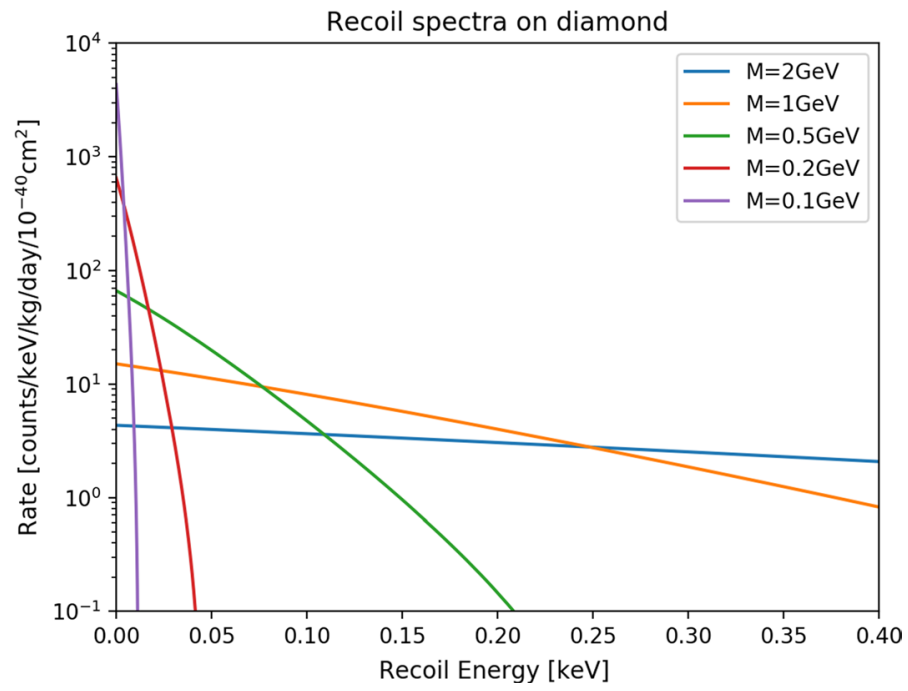

Fig. 1 Expected differential recoil rate in a diamond detector. Different curves are for different masses of DM interacting particle (color figure online) 
diamond as possible qubit for quantum information processing and devices [10]. It is then reasonable to assume that in the next few years diamond will become available and usable for a larger market, especially for scientific applications.

\section{Detector Realization}

We operated a diamond detector of size $(5 \times 5 \times 2) \mathrm{mm}^{3}$, produced by AuDiaTec [11]. It consists of a single crystal diamond, grown by chemical vapor deposition (CVD) on a substrate of Ir/YSZ/Si. The upper limits on the purity certified by the producer are at the level of $1 \mathrm{ppm}$ for nitrogen and $10^{16} \mathrm{~cm}^{-3}$ for boron.

To measure the phonons produced in the absorber, we instrumented the diamond with a TES, very similar to those used for the CRESST experiment [12].

It consists of a thin film (200 nm thick) of tungsten $\left(0.42 \mathrm{~mm}^{2}\right.$ area), directly evaporated onto the diamond surface. Aluminum phonon collectors were then evaporated partly on top of the tungsten area. They increase the phonon collection area without compromising the heat capacity of the sensor and hence provide an enhanced signal amplitude. The aluminum pads serve also for electrical connection to the readout system via aluminum bond wires (diameter $25 \mu \mathrm{m}$ ). Also, a thin gold film is sputtered onto the diamond surface. It connects to the TES and provides a weak thermal connection to the heat bath via gold bond wires (diameter $25 \mu \mathrm{m}$ ). A heater made of a thin gold film was also directly deposited in proximity of the TES. It has the function of stabilizing the sensor temperature in the optimal operating point and is also used to inject heat pulses for detector energy calibration and detector response linearization. Unfortunately, during the measurement presented in this work, the heater was not fully operational and it was not usable for stabilization and calibration purposes. A picture of the TES on the diamond absorber is shown in Fig. 2.

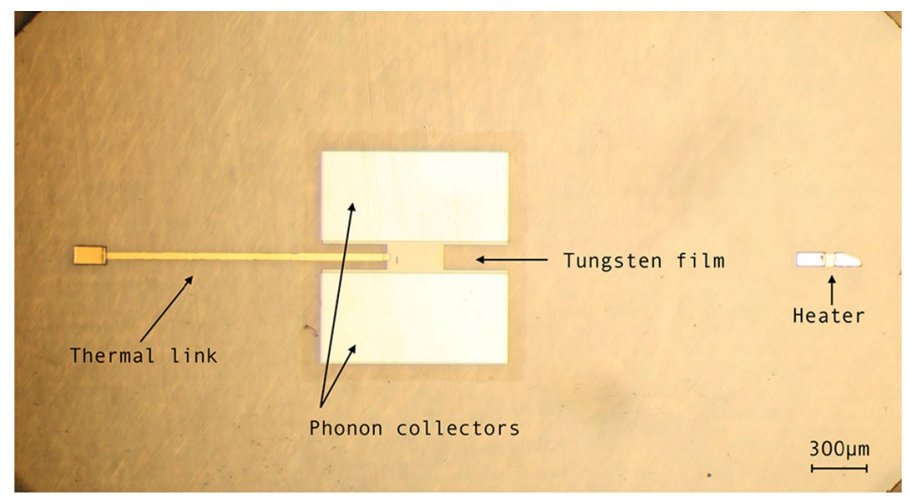

Fig. 2 Picture of the TES fabricated on the diamond absorber. The different components are indicated by arrows. See text for details (color figure online) 
The detector was mounted on a copper holder and held in place on small sapphire balls ( $1 \mathrm{~mm}$ diameter), providing point-like contacts on both top and bottom surfaces.

\section{Results}

The data presented here were acquired in a dilution refrigerator at the MaxPlanck-Institut for Physics in Munich, Germany. The resistance variation of the TES was measured with a SQUID readout system similar to the one used in the CRESST experiment [12].

The cryostat reached a base temperature well below $10 \mathrm{mK}$, and the tungsten TES on the diamond crystal showed a normal-to-superconducting transition centered at about $11 \mathrm{mK}$. The transition curve is shown in Fig. 3. Although not optimized for the instrumentation of the diamond, this is the first transition curve of a tungsten TES operated on a diamond detector that was ever recorded.

A bias current of $4 \mu \mathrm{A}$ was applied to the sensor, and a measurement of a few hours was carried out. The energy spectrum is shown in Fig. $4 .{ }^{55} \mathrm{Fe} \mathrm{X}$-ray source that was placed in proximity of the diamond for the low-energy region calibration shifted out of its position during the detector preparation preventing an accurate energy reconstruction of the acquired events.

It is nevertheless possible to spot a prominent feature in the recorded spectrum of Fig. 4 which we assign to the nominal value $5.9 \mathrm{keV}$ from ${ }^{55} \mathrm{Fe}$. We can then estimate an energy resolution on the baseline of $\sigma=70 \mathrm{eV}$. This preliminary result will be confirmed by a further cryogenic measurement that will be performed in the very near future in the same cryogenic infrastructure. With a fully

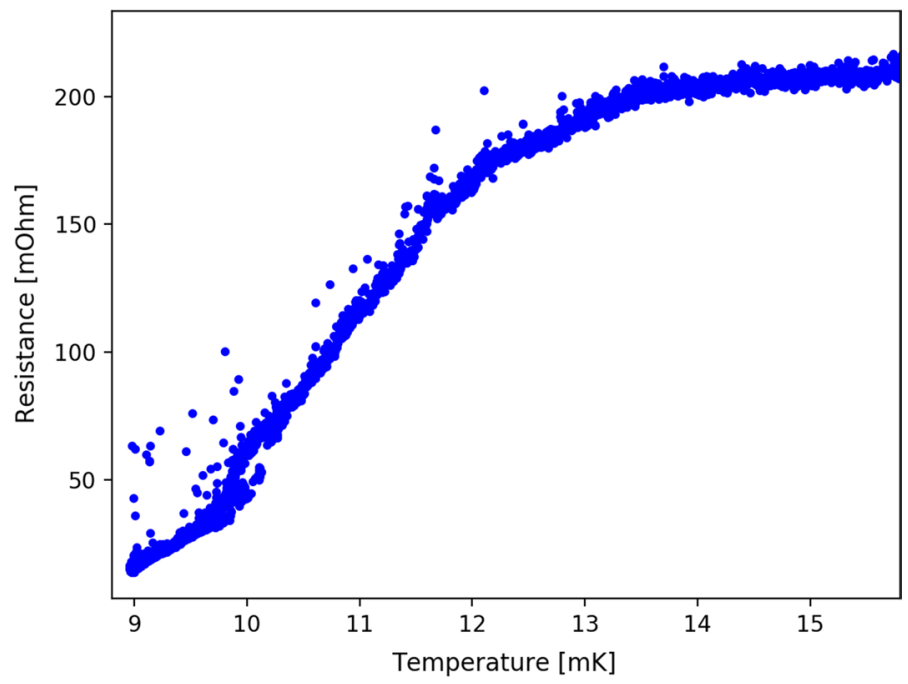

Fig. 3 Normal-to-superconducting transition curve of the TES operated on the diamond detector. The transition in the tungsten film is between 9 and $14 \mathrm{mK}$ (color figure online) 


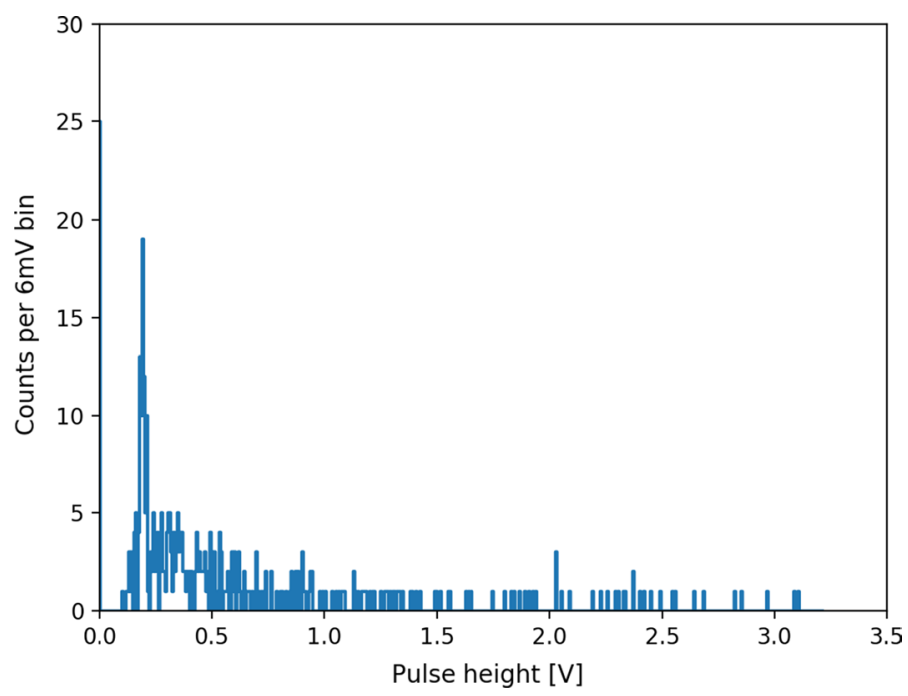

Fig. 4 Uncalibrated energy spectrum acquired with the prototype diamond detector (color figure online)

functioning heater, the sensor will be stabilized in the optimal operating point and the calibration source will provide an accurate energy scale for the evaluation of threshold and energy resolution. The result presented here demonstrates the proof of principle of diamond as cryogenic detector operated with a TES thermometer.

\section{Discussion}

Being clear, the importance of using a light material as target for sub-GeV DM detection, in the following the reach of diamond target for the detection of nuclear recoil DM will be discussed. The standard assumption used to obtain spin-independent limits is a local DM density of $\rho_{\mathrm{DM}}=0.3\left(\mathrm{GeV} / \mathrm{c}^{2}\right) / \mathrm{cm}^{3}$ [13], an asymptotic velocity of $\mathrm{v}=220 \mathrm{~km} / \mathrm{s}$ [14] and an escape velocity of $\mathrm{v}_{\text {esc }}=544 \mathrm{~km} / \mathrm{s}$ [15] (see [4] for more details).

A background level of 3 counts/( $\mathrm{keV} \cdot \mathrm{kg} \cdot$ day $)$ is conservatively assumed, as the one obtained in Ref. [16] for a $\mathrm{CaWO}_{4}$ crystal. However, for a more accurate estimation of the sensitivity in the DM detection landscape, also the background due to the natural radioactive isotopes ${ }^{14} \mathrm{C}$ should be taken into account. This isotope is produced by cosmogenic activation, and it will have an impact on the low-energy part of the spectrum, where the DM signal is expected to appear. ${ }^{14} \mathrm{C}$ is a $\beta^{-}$emitter, with a $Q$-value of $160 \mathrm{keV}$. Nevertheless, it has to be noticed that the use of overburden carbon as starting material for the crystal growth could greatly reduce the amount of the radioactive isotope in the diamond. Concentrations as low as ${ }^{14} \mathrm{C} / \mathrm{C} \sim 10^{-18}$ can be achieved using deep underground methane deposits [8].

In Fig. 5, we calculated the projected sensitivity for an exposure of 1 year. Three different materials are used for comparison: diamond, $\mathrm{CaWO}_{4}$ and germanium. 


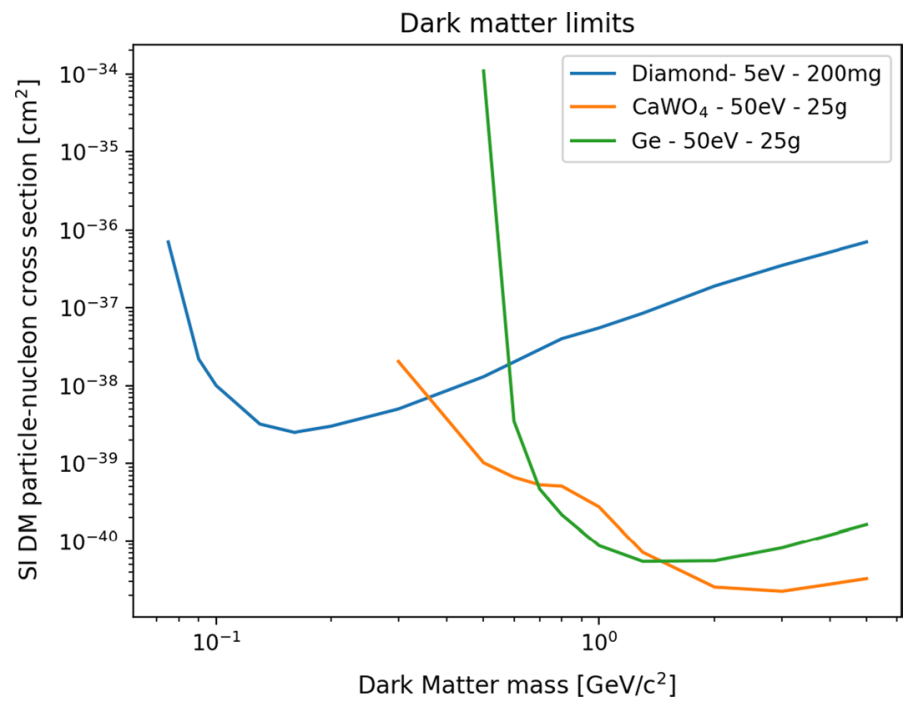

Fig. 5 Sensitivity projections for the elastic, spin-independent DM-nucleus scattering cross section, as a function of the DM particle. Different materials (diamond, $\mathrm{CaWO}_{4}$ and germanium) with different energy thresholds are considered. See text for details (color figure online)

We considered a $(5 \times 5 \times 2) \mathrm{mm}^{3}$ diamond target, and we performed the sensitivity projection assuming a threshold of $5 \mathrm{eV}$. This value that seems achievable based on the state-of-the-art TES technology was derived according to the consideration explained in [7]. For $\mathrm{CaWO}_{4}$ and germanium, we considered a $25 \mathrm{~g}$ target, with a threshold of $50 \mathrm{eV}$ : these values have been successfully demonstrated by the current experimental techniques (see $[4,5]$ for details). Figure 5 demonstrates that even with a limited amount of material, a diamond target would allow to study regions of the parameter space in the sub-GeV range for DM particle that are currently unexplored.

\section{Conclusion}

In this work, we have demonstrated the successful fabrication of a tungsten TES on a CVD single crystal diamond. With a transition temperature of about $11 \mathrm{mK}$, this is the first time that a diamond absorber is operated with a TES sensor as cryogenic detector. This result represents the starting point for the development of a diamond detector which can probe sub-GeV DM candidates.

\section{Acknowledgements Open Access funding provided by Projekt DEAL.}

Open Access This article is licensed under a Creative Commons Attribution 4.0 International License, which permits use, sharing, adaptation, distribution and reproduction in any medium or format, as long as you give appropriate credit to the original author(s) and the source, provide a link to the Creative Commons licence, and indicate if changes were made. The images or other third party material in this article are included in the article's Creative Commons licence, unless indicated otherwise in a credit line to the material. If material is not included in the article's Creative Commons licence and your intended use is 
not permitted by statutory regulation or exceeds the permitted use, you will need to obtain permission directly from the copyright holder. To view a copy of this licence, visit http://creativecommons.org/licen ses/by/4.0/.

\section{References}

1. A. Falkowski, J.T. Ruderman, T. Volansky, JHEP 1105, 106 (2011)

2. K. Petraki, R.R. Volkas, Int. J. Mod. Phys. A 28, 1330028 (2013)

3. K.M. Zurek, Phys. Rep. 537, 91 (2014)

4. A.H. Abdelhameed (CRESST Coll.) et al., submitted to Phys. Rev. D. 100, 102002 (2019)

5. E. Armengaud (EDELWEISS Coll.) et al., Phys. Rev. D 99, 082003 (2019)

6. R. Agnese (SuperCDMS Coll.) et al., Phys. Rev. Lett. 120, 061802 (2018)

7. R. Strauss, J. Rothe, G. Angloher, A. Bento, A. Gütlein, D. Hauff, H. Kluck, M. Mancuso, L. Oberauer, F. Petricca, F. Pröbst, J. Schieck, S. Schönert, W. Seidel, L. Stodolsky, Phys. Rev. D 96, 022009 (2017)

8. N. Kurinsky, T.C. Yu, Y. Hochberg, B. Cabrera, Phys. Rev. D 99, 123005 (2019)

9. N. Coursol et al., Nucl. Instrum. Methods A 312, 24-33 (1992)

10. M.W. Doherty, N.B. Manson, P. Delaney, F. Jelezko, J. Wrachtrup, L.C.L. Hollenberg, Phys. Rep. 528, 1-45 (2013)

11. Augsburg Diamond Technology GmbH, https://www.audiatec.de. Accessed 24 Feb 2019

12. G. Angloher, M. Bauer, I. Bavykina, A. Bento, A. Brown, C. Bucci, C. Ciemniak, C. Coppi, G. Deuter, F. von Feilitzsch, Astropart. Phys. 31, 270 (2009)

13. P. Salucci, F. Nesti, G. Gentile, C.F. Martins, Astron. Astrophys. 523, A83 (2010)

14. D. Kerr, F.J. Lynden-Bell, Mon. Not. R. Astron. Soc. 221, 1023 (1986)

15. M.C. Smith et al., Mon. Not. R. Astron. Soc. 379, 755 (2007)

16. R. Strauss, G. Angloher, A. Bento et al., J. Cosmol. Astropart. Phys. 06, 030 (2015)

Publisher's Note Springer Nature remains neutral with regard to jurisdictional claims in published maps and institutional affiliations. 